\title{
Carcinosarcoma de la vesícula biliar: Caso clínico*
}

\author{
Drs. ÓSCAR TAPIA E. ${ }^{1,2}$, JUAN CARLOS ROA S. ${ }^{1,2}$, LILIA ANTONIO P., \\ 1 Departamento de Anatomía Patológica. Facultad de Medicina. Universidad de La Frontera. \\ 2 BIOREN (Scientific and Technological Bioresource Nucleus). \\ Temuco, Chile.
}

\begin{abstract}
\section{Gallbladder carcinosarcoma. Case report}

Gallbladder Carcinosarcoma corresponds to less than $1 \%$ of all malignant tumors of the gallbladder. We report an 82 years old female subjected to an open cholecystectomy with the preoperative diagnosis of acute cholecystitis. The pathological study of the surgical piece showed a malignant tumor with epithelial (carcinoma) and stromal (sarcoma) components. A postoperative CAT scan showed multiple nodular lesions in the peritoneum and omentum. The patient died eight months after the operation.
\end{abstract}

Key words: Gallbladder, carcinosarcoma, cholecystitis.

\section{Resumen}

El carcinosarcoma de la vesícula biliar (CSVB) es una neoplasia maligna con diferenciación bifásica (epitelial y estromal) y que representa menos del 1\% de todas las neoplasias malignas de la vesícula biliar. Presentamos el caso de una mujer de 82 años sometida a una colecistectomía abierta de urgencia en Septiembre de 2007 con diagnóstico de colecistitis aguda. El examen histológico de la pieza operatoria describe una lesión neoplásica maligna compuesta por una mezcla de componente epitelial (carcinoma) y estromal (sarcoma), este último con extensas áreas de diferenciación heteróloga de tipo condroide. Estos tumores presentan un comportamiento agresivo con rápida diseminación local y recurrencia siendo generalmente difícil la realización de cirugías con intención curativa (R0), condiciones que explican el pobre pronóstico asociado al CSVB.

Palabras clave: Vesícula biliar, carcinosarcoma, carcinoma.

\section{Introducción}

Se denomina carcinosarcoma a una lesión neoplásica con diferenciación bifásica, donde tanto el componente epitelial como estromal son de carácter maligno $^{1}$; siendo descrito este tipo histológico en diversas localizaciones anatómicas tales como útero, pulmón, esófago, riñón, y páncreas ${ }^{2-6}$. Landsteiner, en 1907, describe el primer caso de carcinosarcoma en la vesícula biliar (CSVB), existiendo actualmente alrededor de 67 casos reportados en la literatura mundial y representando esta variedad menos del

*Recibido el 22 de Abril de 2009 y aceptado para publicación el 13 de Julio de 2010.

Financiado en parte por la Dirección de investigación de la Universidad de La Frontera.

Correspondencia: Dr. Óscar Tapia E.

Manuel Montt 112. Código Postal 478-1176. Temuco, Chile

otescalona@gmail.com 
$1 \%$ de todas las neoplasias malignas de la vesícula biliar $^{1,7-9}$.

Presentamos el caso de un CSVB con diferenciación condrosarcomatosa en una mujer de 82 años diagnosticado en el Hospital Hernán Henríquez Aravena de Temuco.

\section{Caso clínico}

Mujer de 82 años con antecedentes de hipertensión arterial y diabetes mellitus tipo 2. Consulta en el servicio de urgencia del Hospital Hernán Henríquez Aravena de Temuco en Diciembre de 2008 por cuadro de dolor en hipocondrio derecho, vómitos y sensación febril. La ecotomografía abdominal demostró una vesícula biliar de paredes gruesas e irregulares con múltiples cálculos en su interior el mayor de $2 \mathrm{~cm}$, con vía biliar intra y extra-hepática de estructura conservada; sin otros hallazgos patológicos. El hemograma mostró leucocitosis (11.200 glóbulos blancos) y anemia normocítica normocrómica (30,5\%); los niveles de bilirrubina, amilasemia y pruebas hepáticas resultaron normales. Con los hallazgos clínicos e imagenológicos se plantea el diagnóstico de colecistitis aguda litiásica. Como hallazgo intra-operatorio se describe una vesícula biliar de paredes induradas y adherida al lecho hepático a nivel del cuerpo y cuello con pequeñas formaciones nodulares induradas en epiplón menor y tejido adiposo pericoledociano, realizándose una colecistectomía abierta extendida.

El examen anatomopatológico de la pieza quirúrgica describe una vesícula biliar de $8,9 \mathrm{~cm}$ de longitud por $9,6 \mathrm{~cm}$ de perímetro con serosa opaca e irregular, pared indurada y engrosada de hasta 1,5 $\mathrm{cm}$, mucosa pardusca de patrón nodular con áreas pardo amarillentas y blanquecinas induradas y otras de aspecto hemorrágico (Figura 1). El estudio his- tológico demuestra extensa infiltración de la pared vesicular por neoplasia compuesta por una proporción variable de componente epitelial y estromal maligno; donde el componente epitelial o carcinomatoso esá representado por un adenocarcinoma tubular y micropapilar moderadamente diferenciado que infiltra hasta en la túnica serosa y el componente estromal o sarcomatoso conformado predominantemente por focos de diferenciación heteróloga condrosarcomatosa (Figura 2). Se realizó estudio inmunohistoquímico con citoqueratinas de alto y bajo peso molecular (CK AE3/AE1), vimentina y S-100 mediante técnica de avidina-biotina-peroxidasa para tejidos fijados en formalina e incluidos en parafina y posteriormente revelados y contrastados con diaminobencidina y hematoxilina respectivamente, demostrándose positividad para citoqueratinas (Figura $3 \mathrm{~B}$ ) en el componente carcinomatoso mientras que vimentina y S-100 (Figura 3C y D) resultaron positivas en el componente sarcomatoso de tipo condroide, hallazgos concordantes con el diagnóstico de carcinosarcoma.

La paciente evoluciona sin complicaciones postoperatorias. El estudio posterior con tomografía computada (TC) de abdomen-pelvis demostró múltiples imágenes nodulares, la mayor de $0,9 \mathrm{~cm}$ en relación a peritoneo y epiplón. Al $6^{\circ}$ mes postoperatorio se objetiva una paciente enflaquecida, con ascitis de moderada cuantía e ictericia de piel y mucosas. Fallece al $8^{\circ}$ mes post-operatorio.

\section{Discusión}

El carcinosarcoma representa menos del 1\% de todas las neoplasias malignas de la vesícula biliar, siendo el primer caso descrito por Landsteiner en 1907 y existiendo actualmente alrededor de 67 casos reportados en la literatura mundial ${ }^{7-10}$. Histológica-

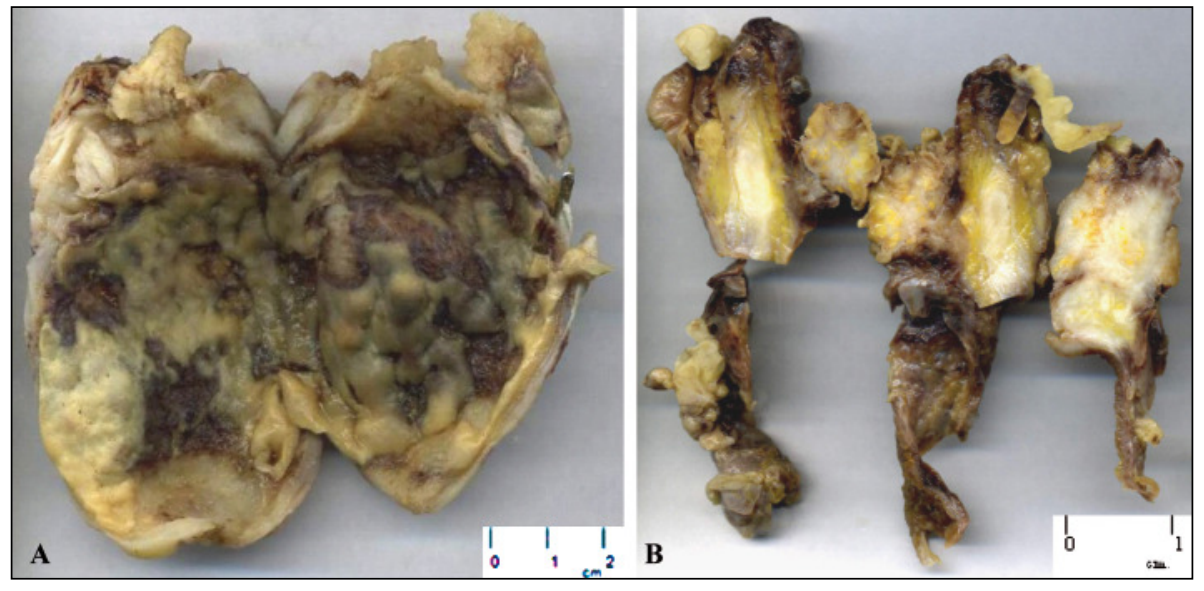

Figura 1. A. Vesícula biliar con mucosa irregular y nodular con áreas pardo amarillentas en partes acartonadas y zonas violáceas de aspecto hemorrágico. B. Superficie de corte, evidencia pared engrosada de hasta 1,5 $\mathrm{cm}$ con áreas blanquecinas irregulares e induradas. 


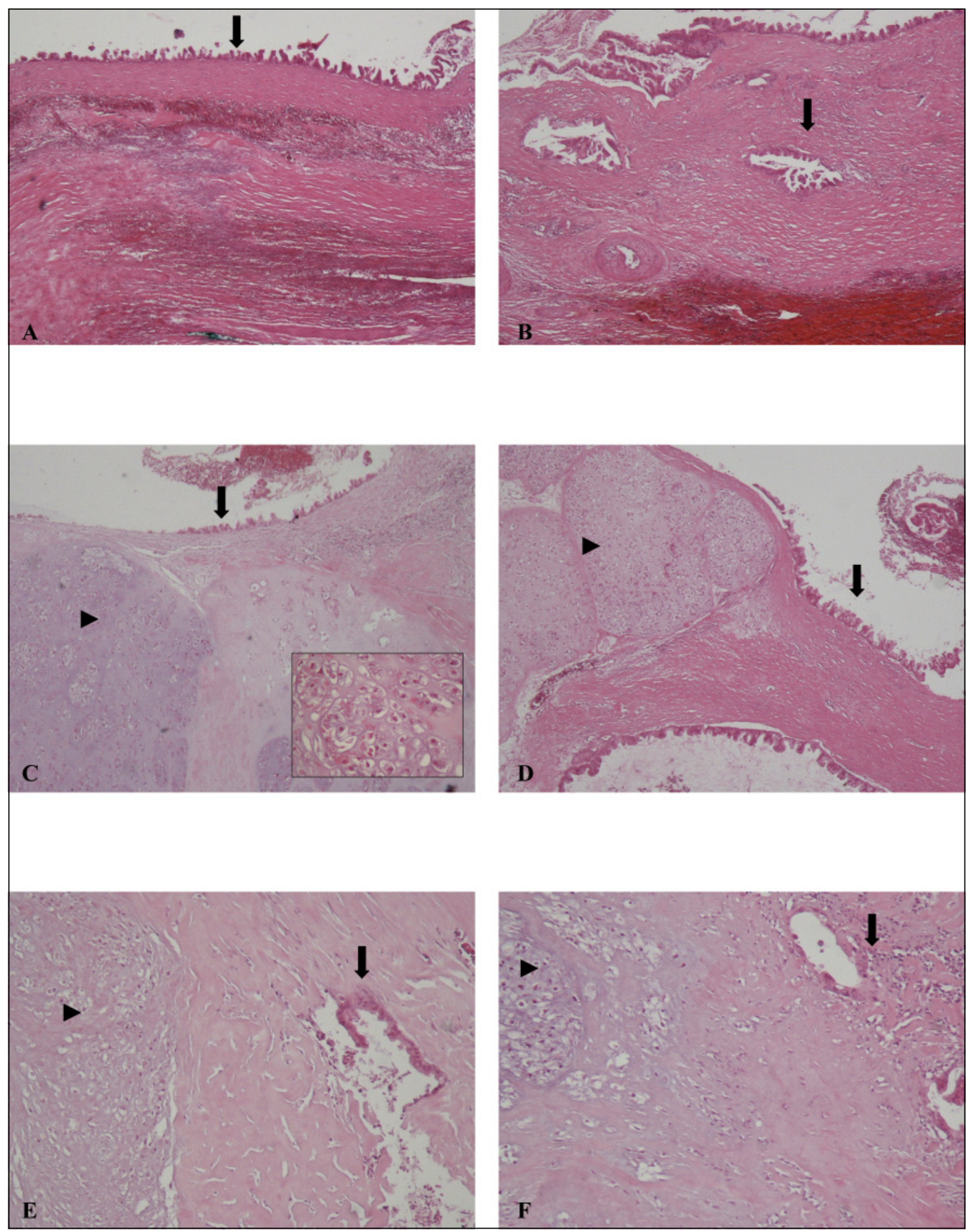

Figura 2. A-D (HE, 40x), E-F (HE, 100x) se reconoce pared de vesícula biliar extensamente infiltrada por lesión tumoral con diferenciación bifásica compuesta por una adenocarcinoma (epitelial) $(\downarrow)$ junto con áreas nodulares basófilas condrosarcomatosas (estromal) ( ). En C (recuadro), se observa con aumento seco mayor (400x) componente estromal conformado por matriz cartilagínea, espacios lacunares ocupados en ocasiones por más de un condrocito, los que presentan marcada atipía nuclear y ocasionales mitosis. 

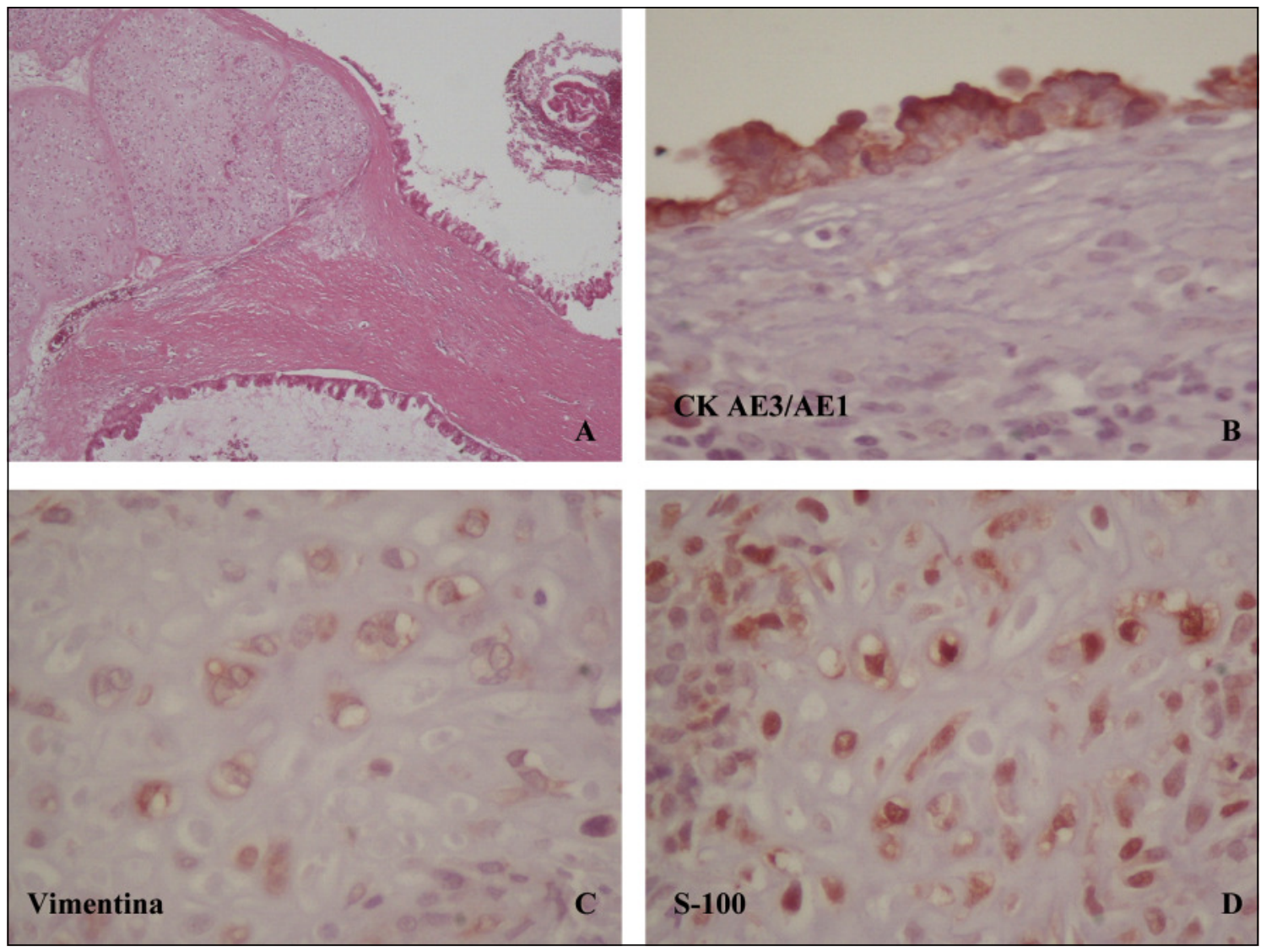

Figura 3. Examen inmunohistoquímico realizado mediante técnica de avidina-biotina-peroxidasa para tejidos fijados, revelado con diaminobencidina y contrastado con hematoxilina expresándose positividad como una coloración pardusca. En B, positividad de patrón lineal y membrana para CK. AE3/AE1 en el componente de carcinoma. En C y D, se aprecia componente condrosarcomatoso con positividad citoplasmática para vimentina y citoplasmática y nuclear para S-100.

mente los carcinosarcomas están compuestos por elementos epiteliales (carcinomatoso) y estromales o mesenquimáticos (sarcomatoso) malignos, por lo que para su diagnóstico se requiere de la presencia y combinación de ambos componentes histológicos. Hasta en el $80 \%$ de los casos de CSVB el componente epitelial es un adenocarcinoma mientras que la diferenciación en el componente estromal es variable, pudiendo estar representado por tejidos homólogos (músculo liso, tejido adiposo) o heterólogos (cartílago, hueso, músculo esquelético) según estos sean tejidos constituyentes mesenquimales propios o no de la vesícula biliar, en nuestro caso observamos un adenocarcinoma tubular y micropapilar con extensas áreas de diferenciación heteróloga condrosarcomatosa, la que está descrita hasta en el $9 \%$ de los $\mathrm{CSVB}^{10}$. Algunas de la teorías propuestas para explicar la combinación de elementos malignos epiteliales y estromales en el carcinosarcoma conside- ran: 1) Teoría tumoral biclonal o por colisión según la cual componente epitelial y estromal proliferan en forma sincrónica; 2) Teoría tumoral monoclonal o por combinación donde ambos componentes derivan de una célula madre en común y 3 ) Teoría por composición o reactiva donde el componente estromal es una proliferación "reactiva" inducida por factores paracrinos derivados del componente epitelial; sin embargo, la evidencia disponible hoy en día por estudios de inmunohistoquímica y citogenética han demostrado el origen monoclonal de esta neoplasia, siendo clasificado actualmente como una neoplasia epitelial maligna y no demostrándose con claridad hasta ahora la importancia pronóstica de la diferenciación estromal homóloga o heteróloga ${ }^{4,10-13}$.

Se ha descrito que este tumor afecta predominantemente a mujeres, en la $6^{\circ}-7^{\circ}$ década de la vida. El diagnóstico se realiza generalmente durante el examen de rutina de la pieza quirúrgica, aunque en caso 
de tumores avanzados estos pueden manifestarse clínicamente por una masa palpable en hipocondrio derecho, dolor abdominal o ictericia y asociado en el $75 \%$ de los casos a litiasis de la vesícula biliar ${ }^{10,14,15}$.

El diagnóstico pre-operatorio generalmente no es sospechado, al igual que en el carcinoma convencional de la vesícula biliar, pudiendo en ocasiones ser sospechado en base a los hallazgos quirúrgicos; pero siendo finalmente el examen histopatológico de la pieza operatoria el que permite confirmar el diagnóstico de cáncer y tipo histológico de este, siendo en ocasiones necesario realizar técnicas de inmunohistoquímica para precisar el diagnóstico o estirpe celular de componentes estromales indiferenciados; en nuestro caso la histología con hematoxilina-eosina permitía reconocer con claridad el componente condrosarcomatoso, confirmándose en el estudio inmunohistoquímico su positividad para vimentina y S-100 al igual que lo publicado por otros autores ${ }^{11,12}$.

Como se mencionó anteriormente, estudios de citogenética han demostrado que el CSVB correspondería a una neoplasia epitelial maligna (carcinoma), por lo que hasta ahora la cirugía es considerada el único tratamiento reconocido para el CSVB, teniendo en cuenta las mismas consideraciones de terapia y pronóstico de acuerdo al nivel de infiltración que en el carcinoma convencional, en el presente caso en base a los hallazgos intra-operatorios sugerentes de una lesión tumoral avanzada se efectuó una colecistectomía extendida, demostrando finalmente el estudio histopatológico infiltración tumoral hasta la serosa vesicular con compromiso del borde quirúrgico cístico. Del mismo modo también en estos pacientes, la efectividad de tratamientos complementarios con quimioradioterapia no ha sido demostrada ${ }^{1,10-12,15}$.

Los CSVB son generalmente diagnosticados en estadios avanzados además de presentar estos tumores altas tasas de recurrencia local y metástasis sistémicas, condiciones que le confieren un pronóstico desfavorable a estos pacientes; donde el promedio de meses de supervivencia reportado es de 5 meses con un rango de 0 a 85 meses y una tasa de supervivencia en el primer año de $19 \%{ }^{10-12,15}$.

Dada la alta prevalencia de cáncer de vesícula biliar en nuestro país, es importante que el anatomopatólogo esté entrenado en reconocer esta entidad, debido al comportamiento agresivo y pobre pronóstico asociado. Algunos trabajos de revisión han intentado evaluar la asociación de factores clínicos y morfológicos con la supervivencia, sin embargo, la heterogeneidad de la información dificulta su interpretación y por otro lado, su baja frecuencia impide la realización de estudios prospectivos de pronóstico $\mathrm{y}$ terapia que permitan mejorar la supervivencia de pacientes con $\mathrm{CSVB}^{10-12}$.

\section{Referencias}

1. Albores-Saavedra J, Henson DE, Klimstra DS. Tumors of the Gallbladder, Extrahepatic Bile Ducts, and Ampulla of Vater. Series 3 Fascicle 8. Washington, DC: Armed Forces Institute of Pathology 2000: 130-3.

2. Silverberg SG, Kurman RJ. Tumors of the uterine corpus and gestational trophoblastic disease. Atlas of tumor pathology, $3^{\text {rd }}$ series, fascicle 3. Washington, DC: Armed Forces Institute of Pathology; 1992. p. 166-77.

3. Zarbo RJ, Crissman JD, Venkat H, Weiss MA. Spindlecell carcinoma of the upper aerodigestive tract mucosa: an inmunohistologic and ultrastructural study of 18 biphasic tumors and comparison with seven monophasic spindle-cell tumors. Am J Surg Pathol. 1986; 10:741-53.

4. Iwaya T, Maesawa C, Tamura G, Sato N, Ikeda K, Sasaki A, et al. Esophageal carcinosarcoma: a genetic analysis. Gastroenterology 1997;113:973-7.

5. Reuter VE. Sarcomatoid lesions of the urogenital tract. Semin Diagn Pathol. 1993;10:188-201.

6. Higashi M, Takao S, Sato E. Sarcomatoid carcinoma of the pancreas: a case report with immunohistochemical study. Pathol Int. 1999;49:453-6.

7. Landsteiner K. Plattenepithelkarzinom und Sarkom der Gallenblase in einem falle von cholelithiasis. Z Klin Med. 1907;62:427-33.

8. Eriguchi N, Aoyagi S, Hara M, Hashino K, Imamura M, Sato S, et al. A. A so-called carcinosarcoma of the gallbladder in a patient with multiple anomalies--a case report. Kurume Med J. 1999;46:175-9.

9. Kazuyoshi N, Masazumi T. Undifferentited spindle cell carcinoma of the gallbladder. a clinicopathologic, inmunohistochemical and cytometric study of 11 cases. Hum Pathol. 1993; 24:1298-1305.

10. Zhang L, Chen Z, Fukuma M, Lee LY, Wu M. Prognostic significance of race and tumor size in carcinosarcoma of gallbladder: a meta-analysis of 68 cases. Int $\mathrm{J}$ Clin Exp Pathol. 2008;1:75-83.

11. Ajiki T, Nakamura T, Fujino Y, Suzuki Y, Takeyama $\mathrm{Y}, \mathrm{Ku} \mathrm{Y}$, et al. Carcinosarcoma of the gallbladder with chondroid differentiation. J Gastroenterol. 2002;37:96671.

12. Okabayashi T, Sun ZL, Montgomey RA, Hanazaki K. Surgical outcome of carcinosarcoma of the gall bladder: a review. World J Gastroenterol. 2009;21:4877-82.

13. Kubota K, Kakuta Y, Kawamura S, Abe Y, Inamori $\mathrm{M}$, Kawamura $\mathrm{H}$, et al. Undifferentiated spindle-cell carcinoma of the gallbladder: an immunohistochemical study. J Hepatobiliary Pancreat Surg. 2006; 13:468-71.

14. Born MW, Ramey, WG, Ryan SF, Gordon PE. Carcinosarcoma and carcinoma of the gallbladder. Cancer Indonesia 1984;53:2171-77.

15. Al-Sheneber IF, Jaber T, Huttner I, Arseneau J, Loutfi A. Carcinosarcoma of the gallbladder: A case report and review of literature. Saudi J Gastroenterol. 2002;8:22-4. 